\title{
A critical analysis of survival in pulmonary arterial hypertension
}

\author{
Dermot S. O'Callaghan* and Marc Humbert", ${ }^{\#,+}$
}

\begin{abstract}
Pulmonary arterial hypertension (PAH) is a rare disease characterised by a progressive increase in pulmonary vascular resistance, leading to right heart failure and premature death. Over the last two decades, a better understanding of the pathogenesis of PAH has led to the approval of several targeted therapies that confer improvements in patients' clinical and haemodynamic status, quality of life and survival. Analysis of recent survival data in PAH cohorts have been biased by the inclusion of prevalent patients included months or years after PAH diagnosis ("survivors") who have a better prognosis than corresponding incident patients diagnosed at the time of recruitment. A critical analysis of recent multicentre incident cohorts has shown survival improvements in the current management era. However, idiopathic PAH remains a severe disease and still carries a poor prognosis. Modern survival figures also highlight the relevance of lung transplantation in eligible PAH patients who are refractory to current best standard of medical care.
\end{abstract}

\section{KEYWORDS: Cohorts, pulmonary arterial hypertension, registry, survival}

$\mathbf{P}$ ulmonary hypertension (PH) encompasses a heterogeneous group of disorders defined by a resting mean pulmonary arterial pressure $(\mathrm{Ppa})$ of $\geqslant 25 \mathrm{mmHg}$, as confirmed by invasive haemodynamic measurements [1]. There are five distinct categories of $\mathrm{PH}$, grouped according to pathophysiological characteristics, expected responses to treatment and prognosis [1,2]. The first of these groups, pulmonary arterial hypertension $(\mathrm{PAH})$, refers to a clinical condition characterised by the finding of pre-capillary $\mathrm{PH}$ (i.e. pulmonary capillary wedge pressure $(P \mathrm{pcw}) \leqslant 15 \mathrm{mmHg})$ at right heart catheterisation. PAH may be idiopathic, heritable, induced by drug or toxin exposure, or develop as a consequence of different disorders, such as connective tissue diseases, HIV infection, congenital heart diseases or portal hypertension. Group 2 refers to $\mathrm{PH}$ associated with a chronically elevated P pcw (i.e. $>15 \mathrm{mmHg}$ ) due to left heart disease. Patients with $\mathrm{PH}$ that develops as a consequence of chronic obstructive or restrictive hypoxaemic lung disease, and those living at high altitude, fall into group 3 of the classification system. Group 4 represents chronic thromboembolic

$\mathrm{PH}$ and group 5 comprises patients with $\mathrm{PH}$ with unclear and/or multifactorial underlying mechanisms.

$\mathrm{PAH}$ is a rare disorder, with an estimated prevalence of $15-50$ patients per one million of the population $[3,4]$. The pathophysiological hallmarks of PAH include endothelial cell dysfunction, endothelial and pulmonary artery smooth muscle cell proliferation, pulmonary vasoconstriction and in situ thrombosis, which lead to a sustained increase in pulmonary vascular resistance $[1,5]$. There are two main components of PAH medical therapy. "Conventional therapies" include oral anticoagulants, diuretics and oxygen, in addition to general measures to help alleviate symptoms. The last two decades have also witnessed the successful development of a number of "targeted therapies" that variously modulate endothelial dysfunction by acting on the prostacyclin, endothelin, and nitric oxide pathways $[1,6]$. Indeed, the advent of the prostacyclin analogue, endothelin receptor antagonist and phosphodiesterase type-5 (PDE-5) inhibitor classes of therapy have been associated with an improvement in prognosis for PAH patients [7].

For editorial comments see page 171.

Some of the results of this article have been previously presented at the 4th International meeting on Pulmonary Rare Diseases and Orphan Drugs, Milan, Italy, February 25-26, 2011.

\section{AFFILIATIONS}

${ }^{*}$ Centre for Lung Health, Mater Misericordiae University Hospital, Dublin, Ireland.

\#Université Paris-Sud 11, Faculté de Médecine

- Service de Pneumologie et Réanimation Respiratoire, Hôpital Bicêtre, AP-HP, Centre National de Référence de l'Hypertension Pulmonaire Sévère, Le KremlinBicêtre, and + INSERM U999, Hypertension Artérielle Pulmonaire, Physiopathologie et Innovation Thérapeutique, Le Plessis-Robinson, France.

\section{CORRESPONDENCE}

M. Humbert

Service de Pneumologie

Hôpital Bicêtre

78 rue du Général Leclerc

94270 Le Kremlin-Bicêtre

France

E-mail: marc.humbert@bct.aphp.fr

Received:

May 202012

Accepted:

June 062012

PROVENANCE

Submitted article, peer reviewed. 
However, a cure for the disease remains elusive and there remains an urgent need for more effective therapeutic approaches [8].

\section{THE EPIDEMIOLOGY OF PAH IN FRANCE}

Regarding patients in group 1 (PAH) of the $\mathrm{PH}$ classification system, data from the French National Pulmonary Hypertension registry confirmed a prevalence rate of idiopathic PAH (IPAH) of six cases per one million of the population and 15 cases per one million for associated causes of PAH [3]. During a 1-yr period between 2002 and 2003, investigators identified 674 confirmed PAH patients in France. Cases of idiopathic, familial, anorexigen, connective tissue diseases (the majority of cases due to systemic sclerosis), congenital heart diseases, portal hypertension and HIV-associated PAH accounted for 39.2, 3.9, 9.5, $15.3,11.3,10.4$, and $6.2 \%$ of the PAH population, respectively [3]. A distinct female predominance was confirmed overall, with the diagnosis of idiopathic and heritable PAH established twice as frequently in females compared to males [1,3]. An even greater proportion of PAH related to anorexigen exposure or associated with autoimmune disease (systemic sclerosis, systemic lupus erythematosis and mixed connective tissue disease) was shown in females [1, 3]. Although most cases of PAH are diagnosed between the third and fifth decades of life, the disorder affects all age groups $[1,3]$. The phenomenon of genetic anticipation (such that PAH diagnosis is established at a younger age in subsequent generations) has been shown in familial PAH, with an increased risk of cases occurring in children and young adults [9].

The clinical manifestations of $\mathrm{PAH}$ are non-specific but progressive, with the majority of patients presenting with worsening dyspnoea that has usually been attributed to other, more common cardiorespiratory disorders [1, 3]. As a consequence, diagnostic delay is usual, with $75 \%$ of patients reporting New York Heart Association functional class (NYHA-FC) III or IV symptoms by the time the diagnosis is established [3]. In an effort to diagnose $\mathrm{PAH}$ at an earlier stage, many specialised centres have established screening programmes for patients that are at high risk (e.g. systemic sclerosis) [10]. In addition, the establishment of Le Plan Maladies Rares (Rare Diseases Strategy) in France led to the creation of a national referral centre (Service de Pneumologie, Hôpital Bicêtre, Assistance Publique Hôpitaux de Paris, Université Paris-Sud, Le KremlinBicêtre, France), to which more than 20 competent regional centres are linked [11]. This strategy has led a greater national awareness of the disease, such that patients throughout the country are more readily identified and referred to specialised centres for optimal management according to international guidelines. It is noteworthy, however, that the initial published data from the French registry highlighted a significant delay before the diagnosis was established, such that the interval between onset of symptoms and confirmation of PAH by right heart catheterisation was on average 27 months. Furthermore, significant variations in the prevalence rates were also observed across different regions of France, ranging from five to 25 cases per one million inhabitants, despite the relative homogeneity of the population. This suggests that in many cases the disease remains undetected or that these individuals diagnosed with $\mathrm{PAH}$ are still not referred in a timely fashion to specialised centres [3].

\section{NATURAL HISTORY OF IPAH ACCORDING TO THE US NATIONAL INSTITUTES OF HEALTH REGISTRY}

The US PH registry was established in the early 1980s with the support of the National Institutes of Health (NIH; Bethesda, MA, USA) [12, 13]. Data from this resource, most commonly referred to simply as the "NIH registry", not only highlighted the burden of this orphan disease in North America but also provided important information regarding its natural history prior to the introduction of targeted therapy [13]. Clinical information and outcome data were collected across US specialised centres on patients diagnosed with "primary" $\mathrm{PH}$, now referred to as idiopathic or familial PAH according to the updated classification system [2]. The majority of the 187 patients identified across the country were considered incident cases, based on the fact that their inclusion followed initial diagnostic right heart catheterisation $[12,13]$. The remaining $36 \%$ of patients were considered prevalent cases (or "survivors") since the diagnosis had been made prior to the establishment of the registry. As subsequently highlighted by the French registry data, this distinction between incident and prevalent cases is important because survival rates for incident $\mathrm{PAH}$ populations are worse than for corresponding prevalent populations. This is explained by the fact that the sickest patients who are diagnosed at the same time as the surviving patients from a prevalent population (i.e. survivors that are included in registries) would be expected to have a high early mortality rate. This high mortality rate subsequently stabilises over time and would then compare favourably to corresponding incident population survival rates (such as those included in registries), since the latter population will include patients that have severe disease and increased risk of early death. This bias is now well recognised and underscores the importance of separately studying incident and prevalent cases when analysing survival data. Indeed, in the NIH registry, the overall survival for IPAH patients was 2.8 yrs, but was shorter in incident compared with prevalent cases (2.6 versus 3.2 yrs, respectively) [13].

The importance of the NIH registry is that it allowed, for the first time, a systematic evaluation of a sizeable cohort of PAH patients and helped establish the influence of clinical, functional and haemodynamic characteristics on outcome in the context of the aforementioned "conventional" therapies alone [13]. One of the most important findings was the critical influence that baseline haemodynamic parameters exert on outcome. Using data from the registry, the "NIH equation" was devised to predict a patient's probability of survival according to baseline right heart catheterisation values for right atrial pressure ( $P$ ra), mean $P$ pa and cardiac index [13]. This equation has been widely employed as a comparator for subsequently published $\mathrm{PAH}$ survival studies [14-16]. However, investigators from these latter comparator studies have overlooked the fact that the NIH registry consisted primarily of incident patients. As such, the application of the NIH equation may be less useful where cohorts enriched by prevalent cases (who would be expected to have better survival) are concerned. This observation is of particular relevance where randomised controlled drug trials are concerned, as a period of clinical stability and/or a prolonged course of treatment with a first-line targeted agent (in the context of combination therapy studies) is often a prerequisite before a patient is considered for study enrolment. 


\section{IMPROVED OUTCOMES OF IPAH PATIENTS IN THE MODERN TARGETED THERAPY ERA: SURVIVAL DATA IN COHORTS TREATED BY i.v. EPOPROSTENOL}

The first major therapeutic breakthrough in PAH came during the early 1990s [17]. In a randomised control trial of 81 subjects with $\mathrm{PH}$ that corresponded to primary (now idiopathic) or familial PAH, continuous intravenous epoprostenol conferred improvements in clinical, functional and haemodynamic parameters when compared to conventional treatment [8, 17]. A survival advantage was also demonstrated, with eight deaths occurring in the control group compared to none in the epoprostenol group. However, it has been suggested that the two groups were not well matched, since the control group in this study may have had more severe disease, as evidenced by lower values for 6-min walk distance (6MWD) at study enrolment. Even so, subsequent analyses of cohorts of consecutive patients in both US and European expert centres confirmed the survival advantage afforded by epoprostenol therapy [14,18]. These studies confirmed that the 1-, 3- and 5-yr median survival rates of patients receiving this agent were significantly better than corresponding survival rates derived from matched historical controls receiving conventional therapy or estimated according to the NIH equation.

\section{IPAH SURVIVAL AND TARGETED THERAPY: EVIDENCE FROM RANDOMISED CONTROLLED TRIALS}

The start of the 21st century has coincided with the successful clinical development of a host of specific PAH therapies. Numerous phase III trials have demonstrated that several members of the endothelin receptor antagonist, PDE- 5 and prostacyclin analogue therapeutic classes confer meaningful haemodynamic, clinical and functional improvements when employed in PAH of different aetiologies [1, 6, 19]. Furthermore, there is emerging evidence indicating an incremental benefit when sequential combinations of treatments from different drug classes are used, with additional investigations underway to evaluate "upfront" approaches [1, 6, 19]. In these therapeutic studies, patients' inclusion criteria have naturally tended to focus principally on those with stable disease that are at low risk of death in the 3-6 months following randomisation. Moreover, investigators have tended to use less robust markers of deterioration in the form of composite scores that reflect time to clinical worsening [1]. Indeed, the only study to definitively show a survival benefit with respect to targeted therapy is that of SIMONNEAU et al. [20] in which the effect of the addition of sildenafil (versus placebo) to long-term epoprostenol therapy was assessed. However, it should be noted that a meta-analysis by GALIÈ et al. [19], in which all published randomised controlled trials were evaluated, found that novel PAH specific therapies confer a survival benefit when taken as a group.

Another strategy frequently adopted by researchers has been to compare the 1-, 2- and 3-yr survival rates among patients receiving active treatment in planned extension phases of randomised clinical trials with the estimated survival rates as calculated using the NIH equation [3, 8]. Investigators utilising this approach have generally reported excellent patient outcomes, with survival rates of $\geqslant 90 \%$ at $1 \mathrm{yr}$, and in the region of $80 \%$ at $2-3$ yrs, figures that compare very favourably with those estimated by the NIH equation. However, these analyses also suffer from a major bias, since patients enrolled in these trials are generally "prevalent" cases with known PAH who are commonly clinically stable. These patients will, thus, appear to have excellent survival rates, but only because their estimated survival is based on an equation that was derived in the main using outcome data from incident PAH populations.

\section{THE FRENCH PAH REGISTRY}

Demonstration of a survival bias in prevalent populations

One of the key features of the French PAH registry is the clear distinction between incident and prevalent patient cohorts, for whom management approaches were relatively homogenous in line with published national and international guidelines. In this regard, it is particularly informative to examine the group of patients with $\mathrm{PAH}$ that is idiopathic, heritable or develops as a result of anorexigen exposure, as survival is usually unaffected by comorbidites in these populations [3,21]. In the French PAH registry, 354 patients were enrolled and followed for 3 years, corresponding to 56 incident and 298 prevalent cases. Analyses of these distinct and well-characterised contemporary populations showed that incident patients displayed an indisputably worse overall outcome, confirming a survival bias among prevalent PAH patients [21, 22]. Furthermore, this survival bias is not only evident when incident and prevalent populations are compared; even within prevalent populations, an analysis by quartiles found that patients in whom the diagnosis of PAH was most recently made fared worse when compared to those with a long-standing diagnosis [22].

\section{Improved patient survival compared to the NIH registry and development of a novel equation to predict survival in incident populations treated in the 21st century}

Data from the incident cohort of the French registry showed an improved survival compared to that predicted by the $\mathrm{NIH}$ equation, indicating the prognosis for $\mathrm{PAH}$ patients has improved in the modern management era [21]. From the registry, a cohort of 190 patients was identified, corresponding to the 56 incident cases and 134 prevalent patients who were diagnosed during the 3 yrs immediately prior to study enrolment. The rationale for including only this subset of the prevalent population was to avoid, insofar as possible, the inherent survival bias of prevalent cases, particularly those with longstanding disease. Therefore, the survival estimates from time to diagnosis were adjusted for the delay between diagnosis and study entry, such that patients were only included in the survival analysis from the point of study entry. For example, a patient recruited $1 \mathrm{yr}$ after diagnosis and followed for a further 3 yrs was considered to enter the risk set at 12 months and was censored at 36 months (i.e. 24 months following study entry). The 1-, 2- and 3-yr survival rates of this rigorously defined population were $82.9 \%$ (95\% CI 72.4-95.0\%), 67.1\% (95\% CI 57.1$78.8 \%$ ), and $58.2 \%$ (95\% CI 49.0-69.3\%), respectively, corresponding to an improvement of $10-15 \%$ over that predicted by the NIH equation. By univariate analysis, an improved survival was shown for females, patients with NYHA-FC I and II symptoms, and those with relatively preserved exercise capacity (as assessed by 6MWD) and cardiopulmonary haemodynamic indices (lower Pra and higher cardiac index values) [21]. Interestingly, in contradistinction to the US registry findings, mean $P$ pa was not shown to have prognostic relevance, an observation that supports earlier findings in more contemporaneous populations [18]. 
Multivariable analysis identified female sex, greater 6MWD and higher cardiac output as independent predictors of survival in incident patients with idiopathic, familial or anorexigeninduced PAH [21]. This allowed modelling of survival in this population and generation of a novel risk-predication equation that could better estimate survival than the existing $\mathrm{NIH}$ equivalent [22]. It is important to stress, however, that this newer "French" equation was not devised to be employed as a comparator for clinical studies but rather to emphasise the variable mortality risks among different PAH subgroups.

An important element not factored into the French study of PAH survival was the potential influence of treatment on assessment of future mortality risk. Indeed, studies reporting outcomes of patients treated by chronic intravenous epoprostenol have confirmed that response to therapy is as crucial a prognostic factor as initial clinical and haemodynamic characteristics [14, 15]. Even so, the validity of the French registry equation was confirmed when its application to a prospective cohort of PAH patients enrolled in four randomised controlled trials and their associated extension studies revealed good agreement between equation-predicted and observed survival [23]. Furthermore, data from a recently published German study has similarly demonstrated that treatment responses translate into long-term outcomes in the modern management era [24], confirming the relevance of those therapeutic targets emphasised in recently published guidelines [1].

\section{DEVELOPMENT OF OTHER PROGNOSTIC EQUATIONS TO ESTIMATE OUTCOMES IN PAH PATIENTS: THE CHICAGO, SCOTTISH AND REVEAL EXPERIENCES}

The original US NIH equation was employed to prognosticate for PAH patients from the early 1980s $[12,13]$. However, several newer, more applicable models have recently been developed using data from modern patient cohorts, essentially rendering the NIH equation obsolete. The Pulmonary Hypertension Connection (PHC) registry gathered demographic and functional data and analysed survival of a contemporary cohort of $\mathrm{PAH}$ patients treated in a single centre in Chicago, IL, USA [25]. This registry identified the same haemodynamic variables employed by the NIH equation to derive a novel equation for patients with idiopathic, familial and anorexigen-induced $\mathrm{PAH}$ based on a similar methodological approach. However, the extended study period of the PHC registry included patients managed both before and after the advent of $\mathrm{PAH}$ specific therapies. The reality of the changing therapeutic landscape of the last two decades may, therefore, have potentially influenced the study's survival analysis, since patients recruited in the modern treatment era would be considered to have generally a more favourable prognosis [19].

Whereas the French and PHC registry equations apply only to patients with idiopathic, familial and anorexigen-induced PAH, the registry to Evaluate Early and Long-Term Pulmonary Arterial Hypertension Disease Management (REVEAL) investigators developed an equation that included patients from all subgroups of World Health Organization (WHO) Group I PAH [26]. Because REVEAL gathered data from a larger population, the resulting prediction model also incorporated a larger number of variables as compared to the French and PHC $\mathrm{PAH}$ registry equations. The use of the associated 22-point REVEAL risk scoring system in newly diagnosed $\mathrm{PAH}$ patients has been validated as a useful tool to help predict probability of survival [27].

Because of concerns of a potential lack of generalisability of registry equations derived in other populations, investigators in the UK analysed baseline demographics, functional status and pulmonary haemodynamics to develop a multidimensional risk score that would apply specifically to PAH patients in the UK [28]. The "Scottish Composite Score" (SCS) was derived as a tool to predict prognosis in incident PAH patients by using baseline mortality predictors in 182 incident patients. The eightpoint scoring system employs six baseline variables (age, sex, aetiology, 6MWD, Pra and cardiac output) to categorise patients into different prognostic groups. The clinical utility of the algorithm was also confirmed following validation in an independent cohort of idiopathic and heritable PAH patients [28]. Interestingly, the predictive accuracy of the SCS was shown to be comparable to that of the PHC registry equation and possibly greater than the French registry [3, 25].

An important difference between the cohorts used to develop the PHC registry [25] and REVEAL [26] equations and their French [22] and UK [28] counterparts is that neither of the US investigator groups adjusted for the time delay between diagnosis and study entry in prevalent patients. As such, no attempt was made to remove the potential survivor bias among recruited prevalent patients, a consideration that might impact the performance of the US equations. Even so, the REVEAL investigators sought to develop a tool which could apply to incident and prevalent cases; in this regard, recent analyses indicate that the REVEAL equation performs well in both incident and prevalent populations [27].

\section{CONCLUSION}

Published survival data from the last 30 yrs with respect to IPAH may not be reliable since analyses have tended to focus predominantly on prevalent cohorts that are known to have better survival than incident populations. However, more recent research efforts that have meticulously and rigorously assessed multicentre cohorts indicate that IPAH patients have an overall improved prognosis compared to their historical counterparts. A number of registry equations have been developed and validated using different baseline patient variables from these well-characterised cohorts. Future studies are likely to increasingly focus on the prognostic relevance of changes from baseline in different outcome variables [24]. Despite these significant advances a cure for $\mathrm{PAH}$ remains elusive and survival for patients remains poor, underscoring the urgent need for novel therapeutic options. Accordingly, and as emphasised by international guidelines, patients should be managed in specialised centres by clinicians that are expert in the diagnosis, assessment and follow-up of those with PAH and are familiar with the complexities of its various treatments. It is also important to emphasise that patients who fail to improve or show clinical worsening despite maximal medical therapy have a very poor prognosis and should routinely be assessed for lung transplantation [1].

\section{STATEMENT OF INTEREST}

D.S. O'Callaghan has received fees for speaking and/or funding for attendance at international congresses and/or unrestricted departmental grants from AstraZeneca, Bayer, Boeringher Ingelheim, GlaxoSmithKline, 
Lilly, Novartis and Pfizer. M. Humbert has relationships with drug companies including Actelion, Aires, AstraZeneca, Bayer, Bristol Myers Squibb, GlaxoSmithKline, Merck, Novartis, Nycomed, Pfizer, Stallergènes, TEVA and United Therapeutics. In addition to being investigator in trials involving these companies, relationships include consultancy service and membership of scientific advisory boards.

\section{REFERENCES}

1 Galiè N, Hoeper MM, Humbert M, et al. Guidelines for the diagnosis and treatment of pulmonary hypertension. Eur Respir J 2009; 34: 1219-1263.

2 Simonneau G, Robbins IM, Beghetti M, et al. Updated clinical classification of pulmonary hypertension. J Am Coll Cardiol 2009; 54: Suppl. 1, S43-S54.

3 Humbert M, Sitbon O, Chaouat A, et al. Pulmonary arterial hypertension in France: results from a national registry. Am J Respir Crit Care Med 2006; 173: 1023-1030.

4 Peacock AJ, Murphy NF, McMurray JJ, et al. An epidemiological study of pulmonary arterial hypertension. Eur Respir J 2007; 30: 104-109.

5 Humbert M, Morrell NM, Archer SL, et al. Cellular and molecular pathobiology of pulmonary arterial hypertension. J Am Coll Cardiol 2004; 43: Suppl. 12, 13S-24S.

6 O'Callaghan DS, Savale L, Montani D, et al. Treatment of pulmonary arterial hypertension with targeted therapies. Nat Rev Cardiol 2011; 8: 526-538.

7 Galiè N, Palazzini M, Manes A. Pulmonary arterial hypertension: from the kingdom of the near-dead to multiple clinical trial metaanalyses. Eur Heart J 2010; 31: 2080-2086.

8 Humbert M, Sitbon O, Simonneau G. Treatment of pulmonary arterial hypertension. $N$ Engl J Med 2004; 351: 1425-1436.

9 Sztrymf B, Yaïci A, Girerd B, et al. Genes and pulmonary arterial hypertension. Respiration 2007; 74: 123-132.

10 Hachulla E, Gressin V, Guillevin L, et al. Early detection of pulmonary arterial hypertension in systemic sclerosis: a French nationwide prospective multicenter study. Arthritis Rheum 2005; 52: 3792-3800

11 Le Pavec J, Humbert M. Centres de référence "maladies rares respiratoires" [Reference centres for rare respiratory diseases]. Presse Med 2007; 36: 933-935.

12 Rich S, Dantzker DR, Ayres SM, et al. Primary pulmonary hypertension. A national prospective study. Ann Intern Med 1987; 107: 216-223.

13 D'Alonzo GE, Barst RJ, Ayres SM, et al. Survival in patients with primary pulmonary hypertension. Results from a national prospective registry. Ann Intern Med 1991; 115: 343-349.

14 McLaughlin VV, Shillington A, Rich S. Survival in primary pulmonary hypertension: the impact of epoprostenol therapy. Circulation 2002; 106: 1477-1482.
15 McLaughlin VV, Sitbon O, Badesch DB, et al. Survival with firstline bosentan in patients with primary pulmonary hypertension. Eur Respir J 2005; 25: 244-249.

16 Barst RJ, Galiè N, Naeije R, et al. Long-term outcome in pulmonary arterial hypertension patients treated with subcutaneous treprostinil. Eur Respir J 2006; 28: 1195-1203.

17 Barst RJ, Rubin LJ, Long WA, et al. A comparison of continuous intravenous epoprostenol (prostacyclin) with conventional therapy for primary pulmonary hypertension. The Primary Pulmonary Hypertension Study Group. N Engl J Med 1996; 334: 296-302.

18 Sitbon $\mathrm{O}$, Humbert $\mathrm{M}$, Nunes $\mathrm{H}$, et al. Long-term intravenous epoprostenol infusion in primary pulmonary hypertension: prognostic factors and survival. J Am Coll Cardiol 2002; 40: 780-788.

19 Galiè N, Manes A, Negro L, et al. A meta-analysis of randomized controlled trials in pulmonary arterial hypertension. Eur Heart J 2009; 30: 394-403.

20 Simonneau G, Rubin LJ, Galiè N, et al. Addition of sildenafil to long-term intravenous epoprostenol therapy in patients with pulmonary arterial hypertension: a randomized trial. Ann Intern Med 2008; 149: 521-530.

21 Humbert M, Sitbon O, Chaouat A, et al. Survival in patients with idiopathic, familial, and anorexigen-associated pulmonary arterial hypertension in the modern management era. Circulation 2010; 122: $156-163$.

22 Humbert M, Sitbon O, Yaïci A, et al. Survival in incident and prevalent cohorts of patients with pulmonary arterial hypertension. Eur Respir J 2010; 36: 549-555.

23 Thenappan T, Glassner C, Gomberg-Maitland M. Validation of the pulmonary hypertension connection equation for survival prediction in pulmonary arterial hypertension. Chest 2012; 141: 642-650.

24 Nickel N, Golpon H, Greer M, et al. The prognostic impact of follow-up assessments in patients with idiopathic pulmonary arterial hypertension. Eur Respir J 2012; 39: 589-596.

25 Thenappan T, Shah SJ, Rich S, et al. Survival in pulmonary arterial hypertension: a reappraisal of the NIH risk stratification equation. Eur Respir J 2010; 35: 1079-1087.

26 Benza RL, Miller DP, Gomberg-Maitland M, et al. Predicting survival in pulmonary arterial hypertension: insights from the Registry to Evaluate Early and Long-Term Pulmonary Arterial Hypertension Disease Management (REVEAL). Circulation 2010; 122: $164-172$.

27 Benza RL, Gomberg-Maitland M, Miller DP, et al., The REVEAL Registry risk score calculator in patients newly diagnosed with pulmonary arterial hypertension. Chest 2012; 141: 354-362.

28 Lee W-TN, Ling Y, Sheares K, et al. Predicting survival in pulmonary arterial hypertension in the United Kingdom. Eur Respir J 2012; [Epub ahead of print DOI: 10.1183/09031936. 00196611]. 